This item was submitted to Loughborough's Research Repository by the author.

Items in Figshare are protected by copyright, with all rights reserved, unless otherwise indicated.

\title{
A diagrammatic view of the equals sign: arithmetical equivalence as a means, not an end
}

PLEASE CITE THE PUBLISHED VERSION

http://dx.doi.org/10.1080/14794800802233688

\section{PUBLISHER}

Routledge (@ British Society for Research into Learning Mathematics)

VERSION

AM (Accepted Manuscript)

LICENCE

CC BY-NC-ND 4.0

\section{REPOSITORY RECORD}

Jones, lan. 2019. "A Diagrammatic View of the Equals Sign: Arithmetical Equivalence as a Means, Not an End”. figshare. https://hdl.handle.net/2134/8588. 
This item was submitted to Loughborough's Institutional Repository (https://dspace.lboro.ac.uk/) by the author and is made available under the following Creative Commons Licence conditions.

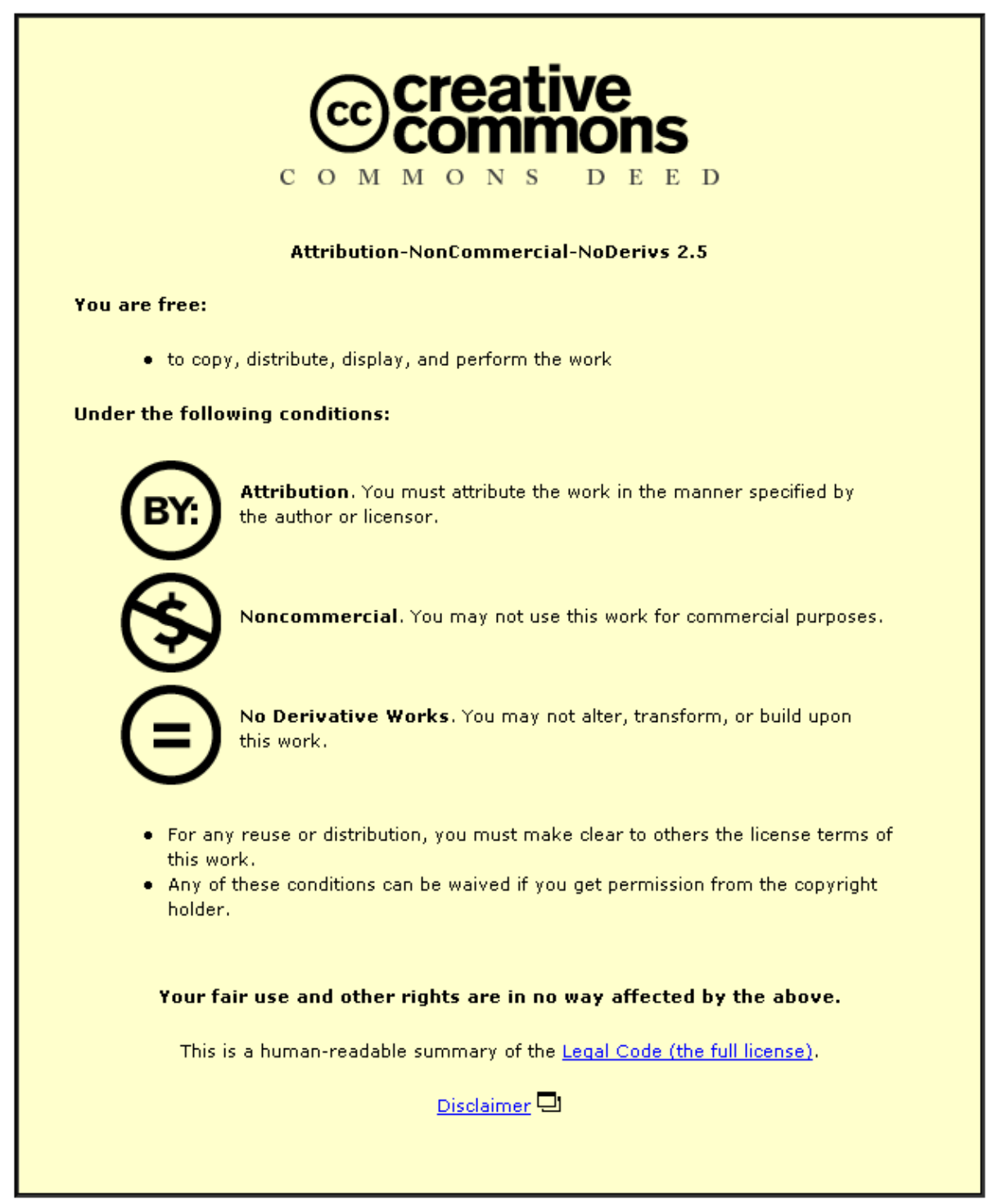

For the full text of this licence, please go to: http://creativecommons.org/licenses/by-nc-nd/2.5/ 


\title{
A diagrammatic view of the equals sign: Arithmetical equivalence as a means, not an end
} Ian Jones

Keywords: equals sign; arithmetic; diagrammatic activity

\begin{abstract}
It is recommended in the mathematics education literature that pupils be presented with equality statements that can be assessed for numerical balance by attending to notational structure rather than computation. I describe an alternative, diagrammatic approach in which pupils do not assess statements but instead use them to make substitutions of notation. I report on two trials of a computer-based task conducted with pairs of pupils and highlight two findings. First, the pupils found it useful to articulate the distinct substitutive effects of commutative ('swap', 'switch') and partitional ('split', 'separate') statements when working on the task. Second, the pupils did not notice that some of the statements presented were in fact false, which suggests their substituting activities were independent of numerical equivalence conceptions. I argue this demonstrates that making substitutions offers task designers a mathematical utility for equality statements that is distinct from, but complementary to, assessing numerical balance.
\end{abstract}




\section{Introduction}

In a world in which $15+28=44$, what can be said about $15+29$ ? In the following scenario, two Year 5 pupils (9 and 10 years) discuss the sum of 15 and 29, having been 'told' that $15+28=44$

Yaaseen: What's 15 add, what's 15 add, er, 29?

Laura: Um, it's 45.

Yaaseen: 45? So we're one off.

Laura: Hmm.

Researcher: How did you work that out so quickly?

Laura: Um, because like that's, um, 15 add $28 .[15+28=44$ is on the computer screen $]$

Researcher: Yeah.

Laura: So just 15 add 29, 28, plus one more is just 45 .

Laura did not view $15+28=44$ as a question to be answered ('is it true?') but as a statement of 'fact' that she transformed in order to infer another 'fact' $(15+29=45)$. In the study reported here, this distinction between presenting arithmetic statements as questions of numerical equivalence and presenting statements as given 'truths' for making transformations of notation is explored.

\section{Pupils' conceptions of equality statements}

In a seminal study that has had a significant influence on subsequent research, Behr et al. (1976) claimed that children view the equals sign as an indicator of computational results rather than as expressing an equivalence relationship. The evidence for this is children's reactions to a variety of presented forms, such as $2+3=7,6=4+10,3=3$ and $2+3=3+2$, in semi-structured interviews. Behr et al. noted a general tendency to impose a computational, left-to-right reading 
on arithmetical statements that conforms to expression = numerals (where expression is any closed string of numerals and operator signs). Even those children who accepted variants, namely numerals $=$ numerals, numerals $=$ expression and expression $=$ expression, attended to a computational reading of calculating expressions and comparing results. Similar results have been found since (e.g. Falkner, Levi and Carpenter 1999; Kieran 1981). This rigid adherence to left-to-right readings leads to a high computational burden for many primary pupils (Gray and Tall 1994) and can become deeply entrenched and resistant to change, persisting well into the later years of schooling (Behr et al. 1976; Kieran 1981; McNeil and Alibali 2005). This impedes later mathematical development, notably when symbolic algebra is introduced into the curriculum (Thomas and Tall 2001). Knuth and others (2006) found that at the start of secondary schooling "a relational view of the equals sign is necessary not only to meaningfully generate and interpret equations but also to meaningfully operate on equations" (309- emphasis in original).

Recent interventionist studies have investigated how pupils' conceptions of the equals sign can be addressed by varying the notation presented in classrooms. For example, Molina, Castro and Mason (2006) focused on the use of computational shortcuts to establish numerical equivalence. They undertook a series of teaching interventions in which statements of various forms, such as $12+11=11+12$, were presented to pupils and reported the emergence of various strategies for shortcutting computation including awareness of commutative and partitional (or de-compositional) properties. Other studies have included notational appeals to generalisation, such as exposing young pupils to statements that include ' +0 ' (Carpenter and Levi 2000), or employing letters to represent unknown amounts (Carraher et al. 2006). Pirie and Martin (1997) reported an intervention in which pupils initially worked with missing number statements of the 
form $\_+\ldots+18=\ldots+53$, and were later presented with similar statements in which the missing numbers were replaced by letters. The intention of these and other studies is that through the careful selection and variation of presented statements pupils will develop conceptions of the general properties of mathematical relations. Such studies have had positive results in broadening pupils' acceptance and understanding of varied statement forms, including those not familiar to them, but the impact on longer term algebraic thinking remains an open empirical question (Carraher et al. 2006; Dörfler 2008; Linchevski and Livneh 1999; Tall 2001).

\section{Diagrammatic activities}

A common thread throughout the interventionist studies described above is the metaphor (implicit or explicit) of the equality statement as a balance with the task goal of establishing whether the notation on both sides represents the same number. The pedagogic rationale is that exposure to a variety of statement examples encourages a conceptual understanding of the mathematical relationships represented by arithmetical notation. This rationale carries an unstated assumption, widespread in mathematics education (Dörfler 2006), that external representations (arithmetical notation in this case) have a perfunctory role as a medium for abstract mathematical ideas. External representations are seen as tools for pupils, teachers and researchers to envisage and communicate otherwise imperceptible, intangible objects. Dörfler in fact argues that this presupposed hierarchy of abstraction over representation is a major factor in many learners' difficulties with mathematics:

"[Pupils] often fail to get close to those genuine objects which mathematics purportedly is all about, they believe they lack the necessary abilities to think 'abstractly', and they are convinced that they do not understand what they are expected to understand. They want to reach through the representations to the abstract objects but without success" (100). 
He argues that mathematics education might be improved by sometimes reversing this hierarchy of abstraction and representation. In the case of written arithmetic this means that numerals and statement forms, rather than numbers and relationships, can become the very objects of learning activities. As Dörfler puts it, symbolic mathematics can be presented to learners as diagrams, or "amalgam[s] composed of an inscription [i.e. an artificial marking on a page or computer screen], a relational structure imposed on it and specific operation rules (including rules for interpretation)"(103). Mathematical domains including arithmetical notation, algebraic equations, graphs, geometrical constructions and so on can be presented as diagrams in this manner. In one sense the term diagram is being used more openly than everyday associations - which might be with 'drawings' rather than 'writings' - would suggest. In another sense, however, it is more restrictive, referring only to those inscriptions that form precise mathematical structures. In the spirit of Peirce (1976), Dörfler refers to learners' constructions, transformations and experimentations with diagrams as diagrammatic activities (or diagrammatic reasoning). These diagrammatic activities provide educators with a view of pupils' mathematics learning as an experimental activity, involving making empirical discoveries about physical inscriptions when transformed (or 'manipulated') according to formal operation rules.

An equality statement presented as a diagram is no longer yet another example for encouraging the development of concepts corresponding to mathematical objects, but instead indicates future possibilities not yet realized. Learners detect patterns in their actions when transforming diagrams, and predict and observe the visual appearance of resultant, modified diagrams. The focus is on actions with inscriptions, mental or physical, rather than exposure to notated examples. Diagrammatic activity is also public and sharable due to its grounding in 
observations (of diagrams) and articulated transformations. This centrality of collaboration and discussion renders mathematics learning observable and concrete to teachers and researchers, and mathematical objects (i.e. physical inscriptions) observable and concrete to pupils. For instructional designers the challenge is to present mathematics as diagrams such that pupils might engage with them in a collaborative and open manner.

When pupils establish the truth status of equality statements of various forms they do of course engage in activities that are to some extent diagrammatic. The above interventionist studies demonstrate pupils articulating the structural properties of notation in reflective and flexible ways when establishing numerical balance. However, the essence of diagrammatic activities, which are based in seeing and articulating potential actions, is curtailed by the task goal of assessing truthfulness. Once a statement has been analysed to a learner's satisfaction, its potential for further activity comes to an end and a new statement must be presented. In the remainder of this paper I describe and report on a task in which pupils used equality statements as tools for further diagrammatic activities, and demonstrate that they did not to attend to the truth or falsity of the statements when doing so.

\section{Arithmetical equivalence as a means}

"The notion of equivalence goes hand in hand with the important mathematical idea of replacement. If two expressions are equivalent then one may be used to replace the other at any time. [Given $3 \times(2 \times 4)=(3 \times 2) \times 4$ then] $3 \times(2 \times 4)$ can replace $(3 \times 2) \times 4$ or, if one finds the counting number equivalent (in this case 24), it can be used to replace the expressions" (Collis 1975, 17).

Collis here seemed to imply that the equals sign can be both a symbol of numerical equivalence and a symbol of interchangeable notation. Similar definitions of a full conception of arithmetical equivalence have been made elsewhere (Freudenthal 1983; Gray and Tall 1994; 
Kieran 1981; Linchevski and Livneh 1999; Skemp 1986). In this section I describe a task in which the equals sign means not 'is the same as' but instead 'can be exchanged for'. Rather than establishing numerical equivalence, the intention is that pupils use arithmetical statements to make substitutions of notation. The proposed 'can be exchanged for' meaning for the equals sign is termed substitutive equivalence here to distinguish it from the numerical equivalence ('is the same as') meaning referred to elsewhere.

The task is computer-based and presents a series of problems, presented to pupils as 'puzzles' to solve, each comprising several arithmetical statements and a single arithmetical term in a box (Figure 1).

Figure 1 here

The software supports two basic functionalities. The first is clicking an equals sign which causes a statement to become highlighted $(8+7=15$ has been highlighted in Figure 1$)$. In this sense the equals sign can be thought of as a handle for taking hold of a statement. The second functionality is clicking numerals and operator signs once a statement has been highlighted. This causes a substitution to take place, or not, as determined by the currently highlighted statement. For example, given the highlighted statement $8+7=15$ in Figure 1, if the inscription $8+7$ in the box is clicked then the outcome will be that shown in Figure 2. This functionality can be considered as a technological embodiment of substitutive equivalence.

Figure 2 here

Substitutions are reversible. If the inscription 15 in the box in Figure 2 is clicked while the statement $8+7=15$ is still highlighted the situation returns to that shown in Figure 1 .

The arithmetical statements, then, are tools that can be used to manipulate the term in the box. Arithmetical statements can also be used to manipulate other statements. For example, if the 
statement $8+7=15$ is still highlighted and the inscription 15 in the statement $15+1=16$ is clicked the latter will become $8+7+1=16$. If the highlighted statement does not determine a substitution for a clicked inscription then nothing happens. For example, clicking the inscription +1 in the box while the statement $8+7=15$ is highlighted will have no effect. The goal for each puzzle presented by the software is to transform the term in the box (referred to as the 'sum' in trials) into a number (referred to as the 'answer' in trials). The inscriptions in the software form a diagram by virtue of the relations determined by the operation rules (interface functionalities) set out above. The software's two functionalities of selecting statements (clicking =) and testing terms (clicking $0,1,2,3,4,5,6,7,8,9$ or + ) allow random experimentation. However, in order to progress and use the software strategically, it is necessary to engage in the diagrammatic activities of iconic matching and substituting. Iconic matching is searching for visually identical inscriptions, and is forced by the embedded operation rule ' $A=B$ acts on occurrences of $A$ or $B$ ' where $A$ and $B$ are any two inscriptions. For example, ,.. Substituting is clicking on an inscription $A$ such that it becomes $B$ (or vice versa) as determined by a highlighted statement $A=B$. For example, $\ldots$ This operation rule provides a formal definition of substitutive equivalence.

Iconic matching and substituting characterise the nature of the observations and manipulations seen to be made by pupils during the trials reported below. At first glance this design rationale perhaps offers a barren vision of notating activity, involving a tiresome procedure of hunting for visual matches and clicking them, with no need for further engagement. Indeed, if one has a clear grasp of the operational rules, this is the most efficient method for completing a puzzle. However, as the data illustrate, learners find the puzzles challenging and motivating (see also Jones 2007b). Furthermore, when working in pairs and encouraged to advise 
one another, learners strive to construct an overall shared sense of the diagrams, and their often surprising models provide insights into mathematical thinking (Jones 2007a).

\section{Background Work and Approach}

Previous trials with pairs of Year 5 children (9- and 10-year-olds) suggested that the software and task promote the diagrammatic activities of iconic matching and substituting (Jones 2007b). It was also found that pupils inferred commutative ('swapping', 'switching') of $a+b=b+a$ statements, and partitional ('splitting', 'separating') readings of $c=a+b$ and $a+b=c$, statements from their distinct transforming effects. The trials reported in this paper build on these findings to investigate whether substitutive equivalence meanings for arithmetical statements are dependent on or independent of numerical equivalence meanings.

The software offers the possibility to explore the relationship between numerical and substitutive equivalence as a result of the operational rule stated above: ' $A=B$ acts on occurrences of $A$ or $B$ ' where $A$ and $B$ are any two inscriptions. It has no bearing whether $A$ and $B$ are conventional arithmetical inscriptions or not, and if they are, whether they are numerically equivalent or not. As such, puzzles can contain false equalities (Figure 3), and yet the term in the box (numerals and operators) in the box can still be reduced to a numeral (without operators) through inscription manipulation.

\section{Figure 3 here}

In the trials reported here, the software was set up to present a sequence of eleven puzzles containing up to nine arithmetical statements. The first two puzzles contained only two compositional statements each and are intended to familiarise learners with operating the software. Puzzle 3 introduces a commutative statement and Puzzle 6 introduces a partitional statement. Note that the terms compositional, commutative and partitional are used here only to 
indicate inscriptions of the forms $a+b=c, a+b=b+a$ and $c=a+b$ respectively. Puzzles 7 to 11 combine compositional, commutative and partitional statements and are increasingly complicated. Puzzles 1 to 7 contain only true statements. Puzzle 8 (Figure 4a) contains the first false statement $(15+28=44)$, which is subtle in that it is only imbalanced by 1. Puzzle 9 (Figure 4b) contains two false equalities. Puzzle 10 (Figure 4c) contains three numerical false equalities, two of which are less subtle than those in Puzzles 8 and 9. Puzzle 11 (Figure 3) contains blatant false equalities and a large computational disparity between the term in the box $(143+77)$ and the 'solution' (23).

Figures $4 a, 4 b, 4 c$ here

These puzzles are slight modifications from those used in the previous trials described above (Jones 2007a; Jones 2007b) and alternative designs would have been possible. First, all statements could have been false equalities from the start, or at least introduced much earlier during the trials. However, judging by previous trials, such a design would not allow the pupils sufficient time to become familiarised with the functionality of the software and nature of the task. Second, the imbalance of false equalities could have been introduced less gradually. For example, the first false equality met, $15+28=44$ (in Puzzle 8), could have been, say, $15+28=1$. However, it was felt initial opportunities should be provided for pupils to notice subtle imbalances as this would be more informative about the degree of their sensitivities to numerical equivalence during the trials. Third, place-value partitions and compositions were used less than in previous trials because their patterns of repeating digits would make them obvious when imbalanced and would not have allowed the gradual introduction of false equalities (e.g. $41=40+2$ compared to $41=27+15)$. 
The generality of the children's activity is not of interest here and no scaling up or attempt to generalise across a statistical population is intended. Neither is learning or knowledge transfer a direct focus. The purpose of the trials was theoretical: to establish the possible outcomes of tasks in which the equals sign means 'can be exchanged for'. As such, the task was artificially polarised to investigate the whether the pupils attended substitutive equivalence as opposed to numerical equivalence. This was not done to find out which meaning for the equals sign is 'best', but to provide a 'window' (Noss and Hoyles 1996) on pupils conceptions of substitutive equivalence in order to analyse them within a research trials context.

\section{Participants, Trial Design and Analysis}

Two trials are reported and commonalities across the data are identified. Both trials took place in economically disadvantaged inner-city areas with English as a second language for the majority of pupils. Trial 1 involved two primary school children, Laura (female, 9 years 11 months) and Yaaseen (male, 10 years 5 months), and lasted a total of 35 minutes. The children had limited classroom exposure to partitional $(c=a+b)$ forms several months prior to the trial and no previous classroom exposure to commutative $(a+b=b+a)$ forms. Laura and Yaaseen were deemed 'mathematically able' by their class teacher ('able' pupils were requested because they were thought to be more likely to to uinderstand the nature of the tasks quickly, and therefore to generate data relevant to the research focus in a single trial). Trial 2 involved two secondary school children, Ajay (male, 12 years 8 months) and Nikisha (female, 12 years 9 months), and lasted a total of 39 minutes. The children had 16 months experience of secondary algebra prior to the trial (excluding school holidays). Their school separates Year 8 children into eight achievement sets and Ajay and Nikisha were in the 'top' set. The disparity in ages between the 
two trails enables the possible effects of classroom mathematics experience on diagrammatic activity to be considered.

The collaborative essence of diagrammatic activities meant single participant trials were not appropriate; at the same time, the exploratory nature of the study meant group or whole class trialing were not appropriate either. As such, paired trials arose as the default design. My role during these trials was to show children how to operate the software, prompt for verbal elaborations (as in 'Why do you think that didn't work?') and offer encouragement and praise.

The composite data of naturalistic discourse and onscreen diagrammatic transformations generated by the task provided a window onto the children's mathematical meaning-making (Noss and Hoyles 1996). These data were captured as screen-movies of the children's interactions along with an audio track of their discussion. The audiovisual movies were then transcribed using the qualitative analysis software package Transana (Woods and Fassnacht 2007). The first stage of analysis involved sifting the data for examples of computational readings (e.g. '7 and 7 make 14' for the inscription $7+7$ ), pattern matching (e.g. 'we need to find another $7+7$ '), commutation (typically using the verbs 'swap' and 'switch') and partition (typically 'split' and 'separate'). Ambiguous cases were not included in the analysis and are not reported here. The second stage, which is of key interest here, involved fine analysis of the children's dialogue when using false equalities to make substitutions, or discussing their possible effects, and in particular looking for evidence of attending to computation and truth assessment.

\section{Findings}

The transcript excerpts presented below are intended to evidence the pupils' (i) diagrammatic activities of iconic matching (searching for visually identical inscriptions) and substituting (describing or predicting transformations); (ii) articulation of commutative and 
partitional meanings for different statement forms; (iii) lack of attention to the truth or falsity of arithmetical statements.

\section{Trial 1}

Year 5: Laura (female, 9 years 11 months); Yaaseen (male, 10 years 5 months)

Laura and Yaaseen were confident and talkative and got to grips quickly with the

functionality of the software during the first two puzzles. After Laura discovered that $15+1=16$ transforms the inscription (15+1) into the numeral (16), thereby completing Puzzle 2, I asked why it had worked ( $\mathrm{R}$ is for 'researcher'). Yaaseen gave a computational explanation (15+1 equals 16) but Laura then offered an iconic matching explanation $(15+1$ is in both the statement and the box):

1. R: Do you know why that one worked?

2. Yaaseen: 'cause that's ...

3. Laura: Oh, 'cause that's 15 add 1 [unclear].

4. Yaaseen: That equals the 16.

5. Laura: Yeah.

6. R: Okay, and what did you say Laura, sorry? [Yaaseen had a louder voice than Laura]

7. Laura: Um, because like that one's 15 add 1.

8. Yaaseen: And that's all the sum.

9. Laura: And that's what it says in the box.

The next evidence for iconic matching coincided with the first evidence for commutative awareness ('the opposite', 'swap', 'switch') during Puzzle 3, which presents 9+1 in the box and the statements $1+9=10$ and $1+9=9+1$.

10. Yaaseen: 9 plus 1, eh? 
11. Laura: Equals 10.

12. Yaaseen: Click the equals and all that first. No, I think you have to get it, that one $[1+9=9+1]$, that's the opposite.

13. R: What's the opposite?

14. Yaaseen: Of this one. 'cause that says 9 plus 1.

15. R: Oh I see what you're saying.

16. Yaaseen: And 1 plus 9.

17. Laura: It's because like that one you have to swap 'em around.

18. R: Sorry, what did you say Laura?

19. Laura: Um, because like that was 9 add 1 and that one's 1 add 9 , um, that one had to be switched round for it, for that to say 1 add 9 .

From then on commutative readings occurred regularly throughout the trial. The children used the word 'swap' a total of 29 times and 'switch' 16 times when referring to $a+b=b+a$ forms or expressing a wish to transform occurrences of $a+b$ into $b+a$.

Evidence for a partitional reading ('separate', 'split') first occurred during Puzzle 6 (box: $30+41$; statements: $41=40+1,70+1=71,30+40=70$ ) which contains the first $c=a+b$ form. Yaaseen inferred a partitional meaning for the statement $41=40+1$ before actually using it.

20. Laura: Um, that box equals 71 .

21. Yaaseen: That's harder.

22. Laura: You need 30 add 41.

23. Yaaseen: 70 , let me try that. Separate them two [40 and 1]. Let me try separating these two. And then ...

24. Laura: Yeah, then that $[30+40=70]$. And then that one $[70+1=71]$. 
25. Yaaseen: Easy! That's easy. It's getting easier and easier.

Throughout the trial the children used the word 'separate' a total of 3 times and 'split' 10 times in reference to the transformational effects of partitioning statements. There was also one occurrence of Yaaseen using the phrase 'split them two back together' when expressing a wish to transform an occurrence of $a+b$ back into its original form $c$. Taken together, these emergent substitutive partitional and commutative readings of $c=a+b$ and $a+b=b+a$ forms provided the children with a powerful strategy for making sense of and solving increasingly complicated puzzles. They started each of Puzzles 7 to 11, which contain compositional, commutative and partitional forms, by identifying $c=a+b$ statements in order to break sums up into constituent elements and then used $a+b=b+a$ and $a+b=c$ statements to reorder and compose these elements. This same strategy was also reported in a previous trial with a different pair of Year 5 children in a suburban school situated in a predominately professional class area (Jones 2007a).

Over the duration of the trial the children's discourse displayed an increasingly sophisticated sense of the onscreen diagrams. The following excerpt illustrates how commutation became a means for enabling further substitutions.

26. Yaaseen: If we can swap them two around.

27. Laura: Yeah.

28. Yaaseen: And swap them with the 33 so we can get the 50 and 11.

29. Yaaseen: Go on, that one.

30. Laura: Huh?

31. Yaaseen: That one.

32. Yaaseen: Now swap them two around. Now you can get 50 add 11. 
Laura and Yaaseen did not comment on the false equalities in Puzzles 8 to 11 (Figures 3, $4 a, 4 b, 4 c$ above). This is not positive evidence that they were oblivious to these numerical imbalances, they may have spotted them and simply not mentioned them. However, two excerpts from the transcript suggest this was not the case. The first is when they were momentarily stuck on Puzzle 8 and Laura gave the incorrect result 45 in response to Yaaseen. Her justification for this result suggests she had not noticed the falsity of $15+28=44$; and Yaaseen's acceptance of her result suggests he had not noticed it either.

33. Yaaseen: What's 15 add, what's 15 add, er, 29?

34. Laura: Um, it's 45.

35. Yaaseen: 45? So we're one off.

36. Laura: Hmm.

37. R: How did you work that out so quickly?

38. Laura: Um, because like that's, um, 15 add 28. [in $15+28=44]$

39. R: Yeah.

40. Laura: So just 15 add 29, 28, plus one more is just 45.

The second observable lack of attention to numerical equivalence occurred during the final puzzle (Puzzle 11, Figure 3) in which many statements are very imbalanced. After working at the puzzle for two and a half minutes, and then getting momentarily stuck, Yaaseen began attending to the computational value of the term, which had so far been transformed from $143+77$ to $50+52+11$. It seems Yaaseen readily accepted that this value can vary without finding this variation peculiar or attributing it to the falsity of the onscreen statements.

41. Yaaseen: 50, you can't, you can't swap 'em round for some reason. Can't, that's the, what's the ans ... 
42. Laura: The answer. So ...

43. Yaaseen: What's ...

44. Laura: 113 ... [i.e. $50+52+11]$

45. Yaaseen: 143 add 77 is a hundred and ... 230. So we need to get to that answer. So far we've got, er, 113. So we're nearly ...

Puzzle 11 is complicated and despite the children's determination and successful emerging strategy they worked on it for a further two minutes before solving it. It was only then, when the box contents $(143+77)$ had been transformed into a single numeral (23), that they finally noticed something amiss with one of the statements $(52+80=23)$.

46. Yaaseen: 80 add 52. Yeah make fift ... ah there! Go on, do that. Then there's, is there eigh ...

47. Laura: 80 add 52.

48. Yaaseen: So you've got to swap them around there.

49. Laura: Yeah.

50. Yaaseen: Then we're done [23 appears in the box]. And that's it. Easy!

51. Laura: Nope. Yeah?

52. Yaaseen: What?! The answer's not 23 is it? Is that right?

53. R: Well, what do you think?

54. Yaaseen: Yeah, 'cause we, that's the wrong answer isn't it? Uh?! 52 add 80 is not 23!

\section{Trial 2}

Year 8: Ajay (male, male, 12 years 8 months ); Nikisha (female, 12 years 9 months)

Ajay and Nikisha were confident and talkative and got to grips immediately with the functionality of the software during the first puzzle. They engaged with the diagrammatic 
activities of iconic matching and substituting quickly with no apparent attention to computational readings. After Ajay efficiently solved Puzzle 3 (box: 1+9; statements: 9+1=10 and 1+9=9+1) with confident guidance from Nikisha, I asked Nikisha how she had known what to do.

55. R: How did you see that Nikisha, because you seemed quite sure.

56. Nikisha: 'cause, erm, the first one, it said 1 plus 9 and on there it said 9 plus 1 and over here it says that they're the same. So if you change that to $m$... to tell the, it's the same thing. You can change that to make the answer.

57. R: Okay. Did you follow that Ajay?

58. Ajay: Yes, I was following it. 1 plus 9 , if you change it to 9 plus 1 . And 9 plus 1 equals 10.

The children completed the first ten puzzles efficiently and confidently taking only ten minutes to do so. Most of their discussion during this time related to the functioning of the software. For example, the following excerpt is from the 25 seconds it took them to complete Puzzle 5 (box: $6+5+3$; statements: $5+6=11 ; 11+3=14 ; 5+6=6+5$ ).

59. Nikisha: You click the equals sign

60. Ajay: Yeah.

61. Nikisha: And then do that one. Then you can make that 11. Click on that equals sign. And then do the 5 plus 6 . And then 11 plus 3 . Equals sign.

For Puzzles 7 to 11, which include compositional, partitional and commutative statements and are increasingly complex, Ajay and Nikisha adopted the same strategy as Laura and Yaaseen from Trial 1. This involved starting with $c=a+b$ statements in order to break sums up into constituent elements and then using $a+b=b+a$ and $a+b=c$ statements to reorder and compose the elements into a final numeral. Unlike Trial 1, however, there was little explicit 
discussion of this strategy and no specific vocabulary to distinguish composing, partitioning and commutating; the pupils instead used the generic term 'change' when referring to substitution. Also, unlike the younger children in Trial 1, their activity was far more based in visual search than substitution making. Their approach, while speedy, was characterised by observation not manipulation, and they rarely highlighted statements or clicked numerals and operators in an exploratory manner.

Despite this, Puzzle 11 (Figure 3) caused them significant difficulties and took a total of eighteen minutes to solve (interestingly, it took the Year 5 children in Trial 1 only four and a half minutes). During this struggle their diagrammatic activity was mainly contemplative but they came up against what they perceived to be dead ends and refreshed the puzzle and started again a total of six times. They also used the term 'swap' a total of 24 times during Puzzle 11 when referring to $a+b=b+a$ statements or expressing a wish to transform occurrences of $a+b$ into $b+a$, despite not having used it at all for Puzzles 1 to 10. Only Nikisha used the term 'split' (two times) to express a wish to transform occurrences of $c$ into $a+b$. Both times this was in reference to using the statement $43+11=65$ to transform 65 back into its original form of $43+11$ rather than in reference to $c=a+b$ statements.

Ajay and Nikisha's discussion displayed their increasingly sophisticated thinking towards the later stages of the trial. The following two excerpts illustrate how they imagined the effects of sequences of substitutions several steps ahead.

62. Nikisha: Might have to make 65 and then [pause] then split it back.

63. Ajay: If you can get 80 first. And then 80 , and if we get 52 and then 52 that will change it to 52 add 80.52 add 80 equals 23 . 
64. R: Something you said a minute ago about if you make the 65 and then split it back. What did you mean by that?

65. Nikisha: Because you couldn't get the positioning right so if you have the two numbers to make 65 which is 11 and 43 and then if you can move the 65 somewhere out and then split it again then you might get it.

The children did not comment on the presence of false equalities in Puzzles 8 to 10 .

Neither did they not notice their presence in Puzzle 11 until they had been working at it for ten minutes and thirty seconds and Nikisha said:

66. Nikisha: Why does it say 50 add 11 equals 80 ?

67. R: What do you mean?

68. Nikisha: 50 add 11 equals 80. [pause] It doesn't. It equals 61 [unclear].

69. R: Had you spotted that before or just now?

70. Nikisha: Before. I don't know why I didn't say it.

71. R: Um?

72. Nikisha: I didn't say it before.

73. R: And is that the only one that's strange?

74. Nikisha: [pause] No there's that one as well. Equals 54. Some of them are right and some aren't.

75. R: And what about on the other puzzles? Are any of, were they all right or were they, were some of those wrong?

76. Nikisha: I don't know. I didn't really look at the sums, to the answers, then. When I asked Nikisha when she had first noticed that $50+11=80$ is wrong she answered 'about one or two minutes [before explicitly saying so]'. However, neither Nikisha nor Ajay 
seemed concerned by the presence of numerical non-equivalences and immediately continued working on the puzzle. During a brief semi-structured interview immediately after the trial I asked what the children made of the presence of such statements.

77. R: What about these sums being wrong. Do you know why that's like that in the puzzle?

78. Ajay: Because it's, um, because the puzzle's different to like adding and stuff like that, yeah? And, um, the puzzle, the aim of it, yeah, is to like get that number and, um. I can't explain it but I know why though.

79. Nikisha: It's about your method. And not about the actually like ...

80. Ajay: Yeah, adding.

\section{Discussion}

The diagrammatic activities of iconic matching (searching for visually identical inscriptions) and substituting (describing or predicting transformations) stand out as a common strand across both trials. In Trial 1 these activities manifested themselves most notably as commutative ('swap', 'switch') and partitional ('separate', 'split') descriptions of the distinct substitutive effects of $a+b=b+a$ and $c=a+b$ (or $a+b=c$ ) statements. In Trial 2 the pupils made fewer distinctions of form during Puzzles 1 to 10 and referred to all transformations simply as 'change'. However, commutative readings became explicit when they struggled for a sustained period during Puzzle 11 and, to a lesser extent, so too partitional readings.

The 'can be exchanged for' meaning of the inscription = was a means rather than an end in two ways. The first is the potentiality of each equality statement to make substitutions. The second is the potentiality of the resultant transformed diagram for making further substitutions (turns 26-32 and 62-65). Such potentialities towards a specified purpose have been termed mathematical utilities within the context of pedagogic task design (Ainley, Pratt and Hansen 
2006). The data suggest that substitutive equivalence, as embodied by the software and task goals, offers a genuine utility for notating tasks.

The key finding is that the pupils distinguished the different transforming effects of statements, but did not articulate their truth or falsity. This suggests that substitutive equivalence meanings were independent of numerical equivalence meanings during the trial. How, then, should we regard the recommendation in the literature that an (implicit or explicit) 'is the same as' meaning should be promoted? Certainly, this recommendation must be heartily endorsed given the strong evidence and persuasive arguments for making it. However, in the light of the findings reported here, it may be that it is incomplete, having been derived from studies in which children analyse statements as an end in itself. As such, an 'is the same as' meaning may have certain limitations for task design, and the claim that such a meaning is necessary (rather than merely sufficient) for understanding and manipulating equations (Kieran 1992; Knuth et al. 2006) is challenged within the context of the trials by the pupils' lack of attention to numerical equivalence when making transformations of notation (turns 33-40; 46-54; 66-76; 77-80). The data presented here offer no direct evidence that the children lacked an 'is the same as' meaning of the equals sign per se as this was not tested, but rather that such a meaning was not explicitly cued during the task.

The recommendation that pupils should be presented with a variety of statement forms must also be endorsed, and indeed was embodied in the diagrams presented by the software. However, as a stand alone design rationale, the variation of form risks promoting a basic relational meaning for the equals sign if pupils simply compute both sides and check the results are equal (Baroody and Ginsburg 1983). Researchers are aware of this and select statements in which internal structure (such as place-value partition) readily reveals numerical balance without 
need for calculation. The recommendation made here is that designers might also consider selecting and presenting statements in terms of their transformational effects, rather than just their internal structure, as a complementary way to draw pupils' attention to form.

\section{Concluding remarks}

It could be countered that the 'can be exchanged for' meaning for the equals sign is problematic because the pupils worked with 'nonsense' puzzles containing errors and therefore their activity was superficial. My argument however is that this disconnect demonstrates that the 'can be exchanged for' meaning is independent of the 'is the same as' meaning. Whereas 'exchanging' has mathematical utility for distinguishing statements by form, 'sameness' has utility for discerning truthfulness. A duality of substitutive and numerical meanings for the equals sign might offer learners novel ways to consider mathematical notation. For example, the inscription $2+4$ appears in both $2+45=47$ and $2+4 \times 5=22$ and yet may not be substituted using $2+4=4+2$ in either case. Furthermore, if robust and flexible, it is speculated that dual meanings for the equals sign might better prepare learners for the transition from arithmetical to algebraic notating at the start of secondary schooling, although this conjecture would require further investigation..

\section{References}

Ainley, Ajay., D. Pratt, and A. Hansen. 2006. Connecting engagement and focus in pedagogic task design. British Educational Research Journal 32: 23-38.

Behr, M., S. Erlwanger, and E. Nichols. 1976. How children view equality sentences (Report No. PMDC-TR-3). Tallahassee, Florida: Florida State University. 
Baroody, A. and Ginsburg, H. 1983. The effects of instruction on children's understanding of the "equals" sign. The Elementary School Journal 84: 198-212.

Carpenter, T. and Levi, Laura. 2000. Developing conceptions of algebraic reasoning in the primary grades. Research Report Madison, WI: National Center for Improving Student Learning and Achievement in Mathematics and Science.

Carraher, D., Schliemann, A., Brizuela, B. and Earnest, D. 2006. Arithmetic and algebra in early mathematics education. Journal for Research in Mathematics Education 37: 87-115.

Collis, K. F. 1975. A study of concrete and formal operations in school mathematics: A Piagetian viewpoint. Victoria, Australia: Australian Council for Educational Research.

Dörfler, W. 2006. Inscriptions as objects of mathematical activities. In New mathematics education research and practice, ed. Ajay. Maasz, and W. Schloeglmann, 97-111. Rotterdam/Taipei: Sense Publishers.

-2008. En route from patterns to algebra: comments and reflections. ZDM Mathematics Education 40: 143-160.

Falkner, K., Laura. Levi, and T. Carpenter. 1999. Children's understanding of equality: A foundation for algebra. Teaching Children Mathematics 6: 232-236.

Freudenthal, H. 1983. Didactical Phenomenology of Mathematical Structures. Dordrecht: Reidel Publishing Company.

Gray, E. M. and D. O. Tall. 1994. Duality, ambiguity, and flexibility: A "proceptual" view of simple arithmetic. Journal for Research in Mathematics Education 25: 116-140. 
Kieran, C. 1981. Concepts associated with the equality symbol. Educational Studies in Mathematics 12: 317-326.

- 1992. The learning and teaching of school algebra. In Handbook of research in mathematics teaching and learning, ed. D. A. Grouws, 390-419. New York: Macmillan.

Jones, I. 2007a. Arithmetical notating as a diagrammatic activity. Proceedings of the British Society for Research into Learning Mathematics 27: 31-36.

Jones, I. 2007b. A logo-based task for arithmetical activity. In Proceedings of the $11^{\text {th }}$ European Logo Conference, ed. I. Kalas. Bratislava, Slovakia. Publisher? Pages?

Knuth, E., A. Stephens, Nikisha. McNeil, and M. Alibali. 2006. Does understanding the equals sign matter? Evidence from solving equations. Journal for Research in Mathematics Education 37: 297-312.

Lima, R. and Tall, D. 2006. The concept of equations: What have students met before? Proceedings of the 30th Conference of the International Group for the Psychology of Mathematics Education 4: 233-241.

Linchevski, Laura. and D. Livneh. 1999. Structure sense: The relationship between algebraic and numerical contexts. Educational Studies in Mathematics 40: 173-196.

McNeil, Nikisha. and M. Alibali. 2005. Why won't you change your mind? Knowledge of operational patterns hinders learning and performance on equations. Child development 76 : 883-899. 
Molina, M., Castro, E. and Mason, Ajay. 2008. Elementary school students' approaches to solving true/false number sentences. PNA: Revista de investigación en Didáctica de la Matemática 2, 75-86.

Noss, R., and C. Hoyles. 1996. Windows on mathematical meanings: Learning cultures and computers. Dordrecht: Kluwer Academic Publishers.

Peirce, C. 1976. The New Elements of Mathematics, Vol. IV, ed. C. Eisele. The Hague: Mouton.

Pirie, S. and Martin, Laura. 1997. The equation the whole equation and nothing but the equation! One approach to the teaching of linear equations. Educational Studies in Mathematics 34: 159-181.

Skemp, R. 1986. The Psychology of Learning Mathematics (2nd ed.). London: Penguin Books Ltd.

Tall, D. 2001. Reflections on early algebra. Proceedings of the 25th Conference of the International Group for the Psychology of Mathematics Education 1: 149-152.

Thomas, M. and Tall, D. 2001. The long-term cognitive development of symbolic algebra. Proceedings of the International Congress of Mathematical Instruction 2: 590-597.

Woods, D, and Fassnacht, C. 2007. Transana v2.20. http://www.transana.org. Madison, WI: The Board of Regents of the University of Wisconsin System.

\section{Figures}




$$
8+7+1
$$

\section{$8+7=15$}

$$
15+1=16
$$

Figure 1: The software used in the study

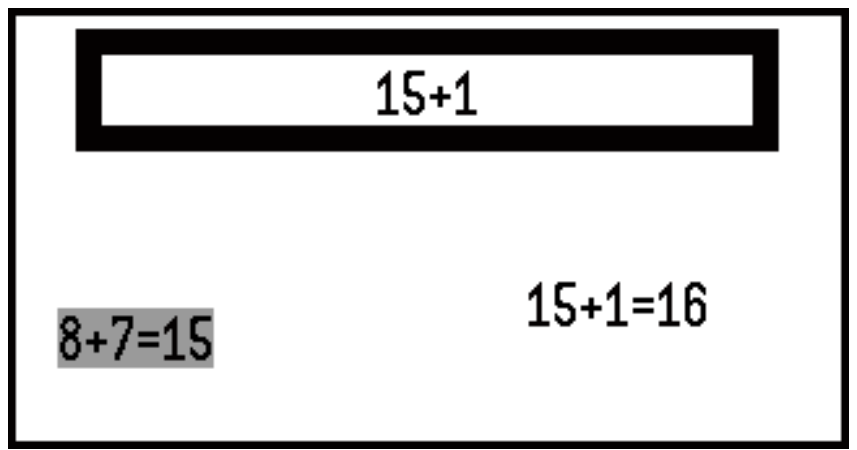

Figure 2: Making a substitution

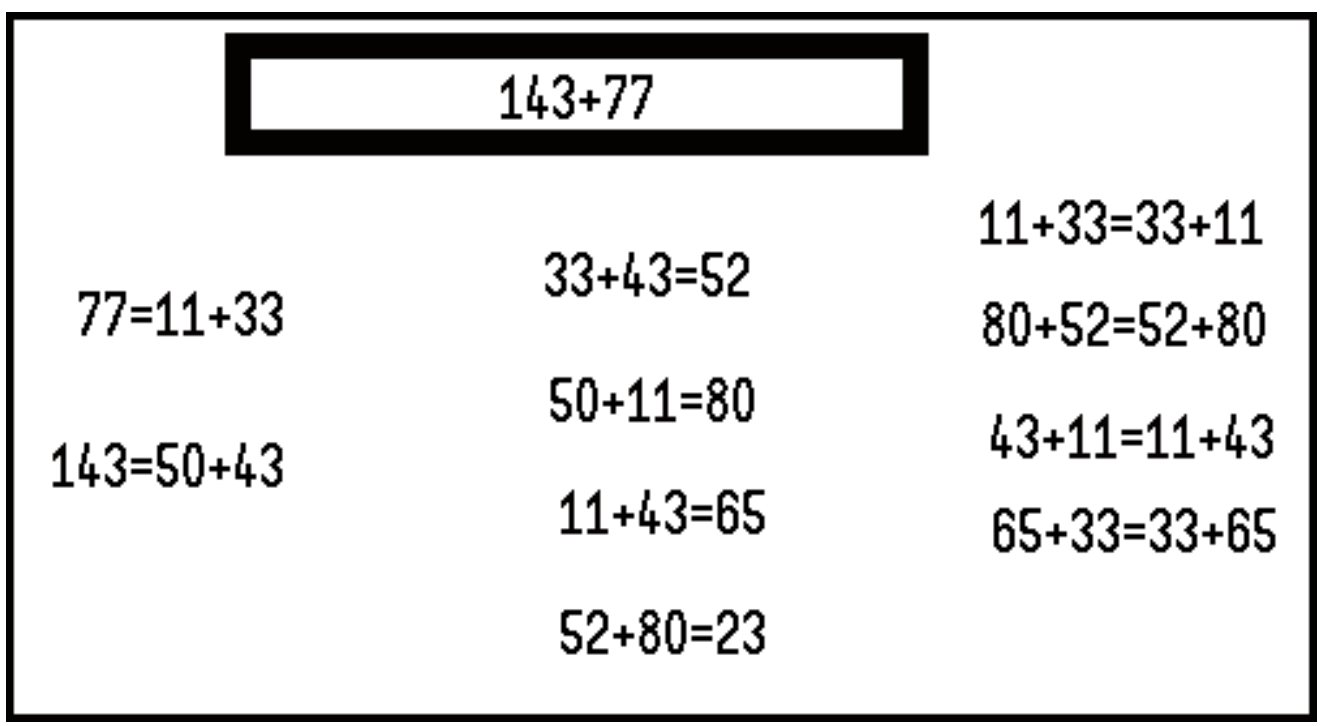

Figure 3: Puzzle containing false equalities 


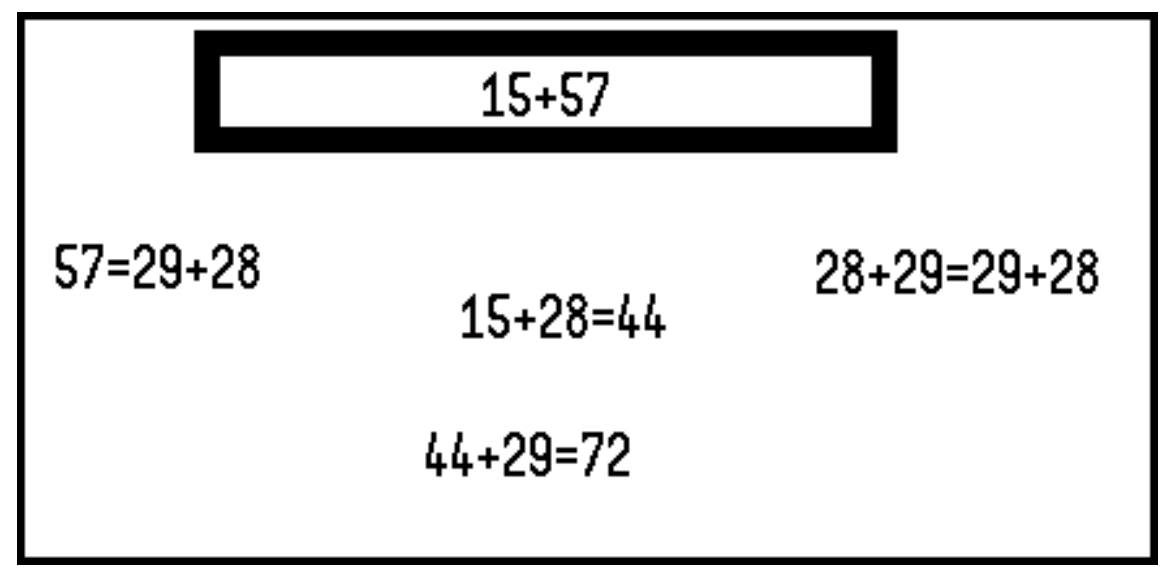

Figure 4a: Puzzle 8

$$
45+37
$$

\begin{tabular}{|c|c|c|}
\hline $37=29+8$ & $29+45=73$ & $73+8=8+73$ \\
\hline $21-\angle 0+0$ & $2 y+45=15$ & $45+29=29+45$ \\
\hline
\end{tabular}

Figure 4b: Puzzle 9

$64+88$

$\begin{array}{lll}64=26+37 & 55+26=81 & 26+69=69+26 \\ 88=33+55 & 33+37=69 & 37+33=33+37 \\ & 69+81=160 & 26+55=55+26\end{array}$


Figure 4c: Puzzle 10 